\title{
Reissner Nordström background metric in dynamical coordinates: exceptional behaviour of Hadamard states
}

\author{
Sukratu Barve and A R Prasanna \\ Theoretical Physics Division, Physical Research Laboratory, Navrangpura, \\ Ahmedabad 380009 , India \\ E-mail: sukratu@prl.res.in and prasanna@prl.res.in
}

Received 7 May 2003

Published 23 February 2004

Online at stacks.iop.org/CQG/21/1505 (DOI: 10.1088/0264-9381/21/6/016)

\begin{abstract}
We cast the Reissner Nordström solution in a particular coordinate system which shows dynamical evolution from initial data. The initial data for the $E<M$ case are regular. This procedure enables us to treat the metric as a collapse to a singularity. It also implies that one may assume Wald axioms to be valid globally in the Cauchy development, especially when Hadamard states are chosen. We can thus compare the semiclassical behaviour with the spherical dust case, looking upon the metric as well as state-specific information as evolution from initial data. We first recover the divergence on the Cauchy horizon obtained earlier. We point out that the semiclassical domain extends right up to the Cauchy horizon. This is different from the spherical dust case where the quantum gravity domain sets in before. We also find that the backreaction is not negligible near the central singularity, unlike the dust case. Apart from these differences, the Reissner Nordstrom solution has a similarity with dust in that it is stable over a considerable period of time. The features appearing in dust collapse mentioned above were suggested to be applicable within general spherical symmetry. The Reissner Nordstrom background (along with the quantum state) generated from initial data, is shown not to reproduce them.
\end{abstract}

PACS numbers: 04.20.Dw, 04.62.+v

\section{Introduction}

The issue of cosmic censorship has several physical implications, two of which have been in focus recently. 
Firstly, it appears that spacetime singularities might signal the visibility of high curvature regions. Even if completely absent in loop quantum gravity [1], classical singularities would correspond to regions of high curvature and nakedness would imply their exposure to asymptotic observers. This itself could lead to observational signatures of particle creation and such effects [2].

Secondly, there appears to be a contrast in the semiclassical behaviour of black holes and naked singularities. That contrast could be carried through into the quantum gravity domain. This has been clearly brought out in the dust collapse case [2]. The covered singularity evaporates leaving behind about a Planck mass before reaching the quantum gravity domain. However, when the initial data correspond classically to naked singularities, most of the star collapses almost intact into the quantum gravity domain all through the semiclassical phase. The star stays almost classical directly till the quantum gravity stage. Moreover, this happens well within the lifetime of the universe [2]. The naked singular solution is thus special from the physical point of view. This argument is subject to the assumption that the behaviour in the dust case is typical of the general scenario.

Out of these, we focus on the second aspect in this paper. Although a contrast in behaviour may still occur, the analysis of dust collapse does not warrant being considered as typical as suggested in [2]. The behaviour of dust depends not only on the semiclassical phase but also on the onset of the quantum gravity domain as well as backreaction considerations. In the spherical dust case, the quantum gravity domain is reached at some stage before the formation of the Cauchy horizon [2] and the backreaction is found to be negligible.

The above behaviour need not always be the case. To this end, we show that the Reissner Nordström metric (charge denoted by $E$ and mass by $M$ ) $E<M$ case is an exception. Before proceeding on that, we first point out that the Reissner Nordström metric can be presented in coordinates which mimic dynamical evolution. As far as we are aware, this has not been done previously. This leads us to two important technical facts. Firstly, one can work with Hadamard states, in principle requiring specification only on the initial Cauchy surface. This justifies the global validity of Wald axioms for the quantum stress tensor which will be assumed in this work. Secondly, we can easily imagine the metric to result from initial data since we have explicit dynamical coordinates. This qualifies the metric to be considered as a collapse leading to a singularity (curvature singularity). The case $E<M$ can be obtained from nonsingular initial data. We then calculate the quantum stress tensor for massless scalar fields on this background and recover the divergence of the quantum stress tensor on the Cauchy horizon obtained earlier [3]. It should be noted that the global validity of Wald axioms is essential in this and similar results concerning the quantum stress tensor. This has not been justified in previous work on the Reissner Nordström metric.

It should be noted that the Reissner Nordström solution is likely to be more of an idealized situation. A realistic collapse would perhaps be accompanied by a radiative influx. Perturbations of the solution are therefore extensively studied especially for instability of the Cauchy horizon [4-7]. Several issues thus arise. Some, like the inflation of the mass function, suggest a non-local characterization of the Cauchy horizon in special models. Others admit observer dependence of divergences. The premise for our theme necessitates consideration of only local quantities, the divergences of which are observer independent. We wish to address the issues in the simplest of cases and present the Reissner Nordström solution as one.

Though we consider the evolution for initial data corresponding to the Reissner Nordström solution, the result is useful as far as a general possibility of an energy burst near Cauchy horizons is concerned. In general, one needs to account for backreaction and invoke quantum gravity to check this. In this example, the $E<M$ initial configuration radiates away right up to the Cauchy horizon. The quantum gravity limit does not occur before the horizon. This 
is unlike the dust case scenario (suggested to be generally valid within spherical symmetry) wherein the quantum gravity domain sets in earlier.

We thus wish to point out that the interplay between physical limits needs to be considered carefully, even within general spherical symmetry. The counter-example of initial data that appears as this simple case, warrants further investigation into the issue.

Apart from this theme, we note that our result for our example does not strengthen the case for a burst because of backreaction. Also, there is a similarity with dust collapse in our example. We find that the configuration does stay intact until it is very close to the Cauchy horizon.

\section{Quantum stress tensor and choice of state}

Much of the physics of quantum fields in curved spacetime is captured by a local quantity, the quantum stress tensor. See for instance chapter 6 of [8] (the central problem). After several approaches to regularize and renormalize this quantity, Wald came up with four axioms which restricted the form of the quantum stress tensor to a very large extent [9].

From the Wald axioms and the trace anomaly in $2 \mathrm{~d}$, one can show that the quantum stress tensor is of the form

$$
\begin{aligned}
& \left\langle T_{u u}\right\rangle=C(1 / C)_{, u, u}+\mathcal{A} \mathcal{A}(u) \\
& \left\langle T_{v v}\right\rangle=C(1 / C)_{, v, v}+\mathcal{B B}(v) \\
& \left\langle T_{u v}\right\rangle=\mathcal{R} / 24 \pi
\end{aligned}
$$

where the line element is expressed in double null coordinates as $\mathrm{d} s^{2}=C^{2} \mathrm{~d} u \mathrm{~d} v$ and $\mathcal{R}$ is the scalar curvature of the background spacetime. The information about the state is contained in $\mathcal{A A}$ and $\mathcal{B B}$. It can easily be argued, based on asymptotic behaviour of $\left\langle T_{\mu \nu}\right\rangle$, that $\mathcal{A} \mathcal{A}$ and $\mathcal{B B}$ are finite in their domain of definition [3].

The important assumption here is that the Wald axioms, especially the conservation of the stress tensor, hold all over the spacetime. Physically, one would like to ensure that only by specifying initial conditions near a Cauchy surface, expecting the equations to hold all through the evolution. Indeed this is possible if one chooses Hadamard states. For a definition and related mathematical development see $[10,11]$. These states can be defined using the singularity structure of the two-point function. They have two useful properties: (a) that they satisfy Wald axioms if they stay Hadamard and (b) by choosing the singularity structure near a Cauchy surface, one is essentially guaranteed that the Hadamard form is preserved all throughout the Cauchy development [12].

In our example, the choice of state is automatically contained in the choice of the $\mathcal{A A}$ and $\mathcal{B B}$. Certainly, there would be choices corresponding to Hadamard states and that poses no conceptual difficulty. However, property (b) requires careful attention since it would restrict considerations to Cauchy developments only. In particular, if the spacetime or region of spacetime under consideration is realized as a Cauchy development then we can assume properties (a) and (b), and thereby we can be assured of the formulae (1) holding all over the region.

We show that the Reissner Nordström solution can be explicitly realized as a time evolution of initial data, justifying our use of Wald axioms in a global sense. In fact, we show that we can recast the metric in coordinates such that the evolution becomes explicit. 


\section{Reissner Nordström solution using special coordinates}

We develop the first part of the analysis in this section, wherein we recast the general Reissner Nordström solution. However, for further analysis, only $E<M$ is suitable.

We consider the field equations for spherical symmetry. In the first subsection we make certain assumptions on the radial pressure and on the gravitational potential. We show in the next subsection that further choice recovers the Reissner Nordström metric. This enables us to cast the metric in a form similar to dynamical evolution. This has been done for the Schwarzschild metric [13]. In the following subsection we show that the condition for nakedness is indeed $E^{2}>M^{2}$. Finally we present the Penrose diagram illustrating the region covered by the coordinates.

\subsection{Spherical symmetry}

The line element in coordinates $(t, r, \theta, \phi)$ is given by

$$
\mathrm{d} s^{2}=e^{\sigma(t, r)} \mathrm{d} t^{2}-e^{\omega(t, r)} \mathrm{d} r^{2}-R^{2}(t, r) \mathrm{d} \Omega^{2} .
$$

We use a source

$$
T_{v}^{\mu}=\operatorname{diag}\left[\rho(t, r),-p_{r}(t, r),-p_{T}(t, r),-p_{T}(t, r)\right] .
$$

The general field equations for spherical symmetry (see, for example, [14] and references therein) are

$$
\begin{aligned}
& 2 m^{\prime}(t, r)=8 \pi \rho(t, r) R^{2} R^{\prime} \\
& 2 \dot{m}(t, r)=-8 \pi p_{r}(t, r) R^{2} \dot{R} \\
& \sigma^{\prime}(t, r)=-\frac{2 \rho^{\prime}}{\rho+p_{r}}+\frac{4 R^{\prime}\left(p_{T}-p_{r}\right)}{R\left(\rho+p_{r}\right)} \\
& \dot{\omega}(t, r)=-\frac{2 \dot{\rho}}{\rho+p_{r}}-\frac{4 \dot{R}\left(\rho+p_{T}\right)}{R\left(\rho+p_{r}\right)}
\end{aligned}
$$

where

$$
2 m(t, r) / R-1=e^{-\sigma} \dot{R}^{2}-e^{-\omega} R^{\prime 2} .
$$

Here $m(t, r)$ is a free function arising out of integration of the Einstein equations. Its initial value $m(0, r)$ is interpreted as the mass of the interior of the shell with coordinate $r$.

In order to proceed towards obtaining the Reissner Nordström metric, we impose certain assumptions within spherical symmetry. These are similar to but more general than those in [14]. We cast them in a particular form so that the generalization is evident,

$$
8 \pi p_{r} R^{2}=\mathcal{A}(r) \mathcal{B}^{\prime}(R)
$$

and

$$
\sigma^{\prime}(t, r)=\psi(r) Q^{\prime}(R) R^{\prime}
$$

From the last assumption above using the fact that the Ricci tensor component $\mathcal{R}_{t r}=0$, we obtain

$$
R^{\prime 2} e^{-\omega}=A(r) e^{Q(R) \psi(r)}
$$

where $A(r)$ is arbitrary.

From the time rate of change of $2 m$, and with the second to last assumption, we get

$$
2 m(t, r)=\mathcal{A}(r) \mathcal{B}(R)+2 m_{o}(r)
$$

where $2 m_{o}(r)$ is arbitrary and $\mathcal{B}(R)=\int \mathcal{B}^{\prime}(R) \mathrm{d} R$. 
Further, from the definition of $2 m$, we obtain, using these results,

$$
\dot{R}^{2} \mathrm{e}^{-\sigma}=\frac{\mathcal{A}(r) \mathcal{B}(R)+2 m_{o}(r)}{R}-1+A(r) \mathrm{e}^{Q(R) \psi(r)} .
$$

This is the evolution of $R$ in time. Given $2 m_{o}(r)$, and using equation (14), we can obtain the 3-metric (and also extrinsic curvature) at any time. Thus we have set up a system of evolution for initial data, much like that in [14].

\subsection{Reissner Nordström solution}

We now show that the Reissner Nordström metric can be cast into the initial value form using certain special cases of the assumptions made.

The metric is usually presented as

$$
\mathrm{d} s^{2}=P(R) \mathrm{d} t^{2}-P(R)^{-1} \mathrm{~d} R^{2}-R^{2} \mathrm{~d} \Omega^{2}
$$

where $P(R)=1-2 M / R+E^{2} / R^{2}$. We perform the following transformation,

$$
\begin{aligned}
& t=T+\int \frac{g(R)}{P(R)} \mathrm{d} R \\
& r=T+\int \frac{1}{g(R) P(R)} \mathrm{d} R
\end{aligned}
$$

where $g$ is chosen so that $P(R) /\left(1-g^{2}(R)\right)>0$ which maintains the signature of the transformed metric.

The metric reduces to the general spherically symmetric form (4) with

$$
\begin{aligned}
e^{\sigma} & =\frac{P}{1-g^{2}} \\
e^{\omega} & =\frac{g^{2} P}{1-g^{2}} .
\end{aligned}
$$

One can easily check that this is recovered from the assumptions of the previous section with the particular choice, $\psi=1$ and $\sigma=Q=\ln P /\left(1-g^{2}\right)$. In order to obtain a solution for $R$ in terms of $t$ and $r$, we choose $A(r)=\mathcal{A}(r)=1$ with $2 m_{o}(r)=2 M$ and $\mathcal{B}(R)=$ $-R P(R)+R-2 M$. Using this in equation (15) we obtain

$$
r-t=\int \frac{1-g(R)^{2}}{P(R) g(R)} \mathrm{d} R \text {. }
$$

The Reissner Nordström metric can now be recast as

$$
\mathrm{d} s^{2}=\frac{P(R)}{1-g(R)^{2}} \mathrm{~d} t^{2}-\frac{g(R)^{2} P(R)}{1-g(R)^{2}} \mathrm{~d} r^{2}-R^{2} \mathrm{~d} \Omega^{2} .
$$

We can choose the function $g$ to be a constant $g_{o}$ with the condition $1-g_{0}^{2}>0$ needed ( $P>0$ for $R>R_{+}$). In case $P$ becomes negative (which it does when $E \leqslant M$ between the roots $R_{-}$and $R_{+}$), we choose $g$ as follows. For the region $R \geqslant R_{+}+\epsilon$, we choose $g(R)=g_{0}$. For $R_{+}+\epsilon \geqslant R \geqslant R_{+}-\epsilon$ we choose $g$ to be a $C^{\infty}$ function $\left(C^{\infty}\right.$ at the endpoints of the interval also). Further $g\left(R_{+}\right)=1$ is chosen with the condition $\lim _{R \rightarrow R_{+}} \frac{P(R)}{1-g^{2}(R)}>0$. For $R_{+}-\epsilon \geqslant R$, we choose $g$ to be a constant $g_{1}>1$.

Additionally, we need to set $\dot{R}<0$ to mimic a collapse scenario. This needs $g>0$. The solution of (21) can now be obtained explicitly $\left(R \geqslant R_{+}+\epsilon\right)$.

$r-t=\frac{1-g_{o}^{2}}{g_{o}}\left[R+2 M / 2 \ln \left(R^{2}-2 M R+E^{2}\right)+\frac{(2 M)^{2}-2 E^{2}}{2}\right] H(R)$ 
where $H$ is defined as

$$
\begin{aligned}
& H(R)=\left(E^{2}-M^{2}\right)^{-1 / 2}\left(\tan ^{-1}\left(\frac{R-M}{\left(E^{2}-M^{2}\right)^{1 / 2}}\right)-\tan ^{-1}\left(\frac{-M}{\left(E^{2}-M^{2}\right)^{1 / 2}}\right)\right. \\
& \quad \text { iff } \quad E^{2}>M^{2} \\
& H(R)=(M-R)^{-1} R / M \quad \text { iff } \quad E^{2}=M^{2} \\
& H(R)=1 / 2\left(M^{2}-E^{2}\right)^{-1 / 2} \ln \frac{1-\frac{R}{M+\sqrt{M^{2}-E^{2}}}}{1-\frac{R}{M-\sqrt{M^{2}-E^{2}}}} \quad \text { iff } \quad E^{2}<M^{2} .
\end{aligned}
$$

For $R \leqslant R_{+}-\epsilon$ (which will be needed in the further sections), we simply replace $g_{0}$ in (23) by $g_{1}$.

For $R<R_{-}$, the case we would need for leading order behaviour of $r-t$ in terms of $R$, we note that $1-g^{2}>0$ (as $P$ is positive again). It is not difficult to see that this behaviour of $r-t$ in terms of $R$ is cubic. One can check explicitly that the coefficients of the linear and quadratic terms in $R$ cancel out in the equation (similar to (23) at leading order) and one obtains the following:

$$
r-t=\frac{1-g(0)^{2}}{g(0)} K R^{3}
$$

where

$$
\begin{aligned}
K & =\frac{1}{3 E^{2}} & \text { iff } & E^{2}>M^{2} \\
K & =\frac{-1}{3 E^{2}} & \text { iff } & E^{2}=M^{2} \\
K & =\frac{M^{2}}{3 E^{4}}\left[11-12 \frac{M^{2}}{E^{2}}-\frac{3 E^{2}}{2 M^{2}}\right] & \text { iff } & E^{2}<M^{2} .
\end{aligned}
$$

Thus $K$ and therefore $r-t$, is positive only for the case $E^{2}>M^{2}$. It is this leading order behaviour that is important for further analysis.

The behaviour of the right-hand side of equation (23) can be easily checked to be monotonic. Thus, the $R=0$ curve is the locus $t-r=0$. It is also easily checked that the Kretschmann scalar diverges when $R=0$. Thus, the singularity occurs when $t-r=0$. Also, the central singularity forms at $(t=0, r=0)$.

Using the monotonicity and the leading order behaviour (27), we note that in the case $E<M$, the singularity is absent for all $t<0$ at $r=0$. We begin with an initial surface $t=t_{\text {in }}$. The initial data evolve from there to form the singularity.

Thus we have cast the case $E<M$ in a manner which enables us to compare with spherical dust collapse.

The $E>M$ case can also be addressed similarly. In this case, one cannot have $t<0$ at all for $r=0$. Thus the surfaces of constant $t$ begin from $t=0$ and thus include the singularity from the beginning.

\subsection{Nakedness of the Reissner Nordström solution}

At this juncture it is easily possible to demonstrate that the singularity is locally naked. It is done in a manner equivalent to [15].

Casting the metric into the usual double null form,

$$
\mathrm{d} s^{2}=P(R) \mathrm{d} u \mathrm{~d} v+R^{2} \mathrm{~d} \Omega^{2}
$$




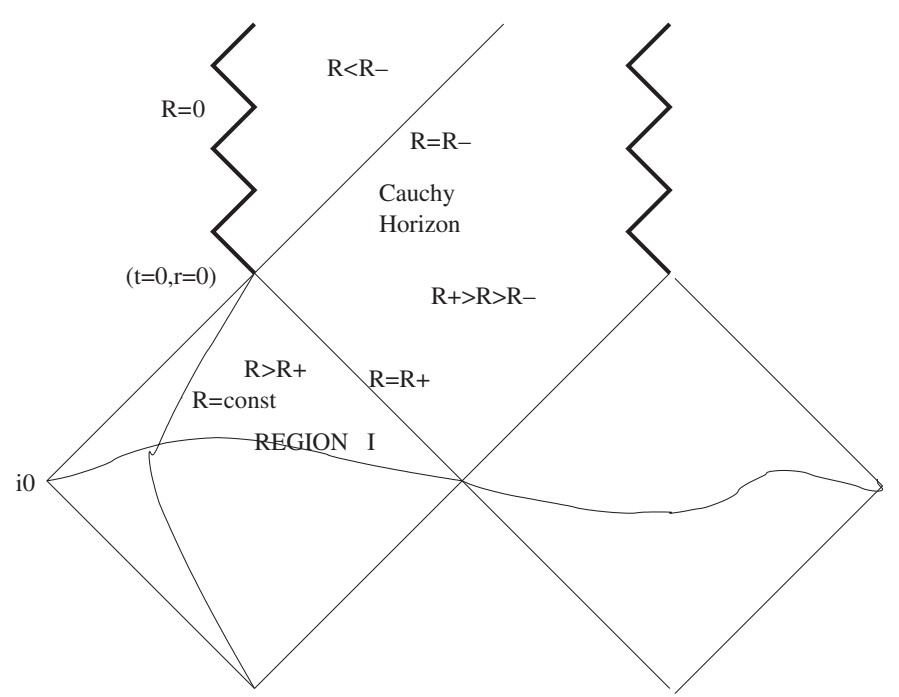

Figure 1. Penrose diagram for the Reissner Nordström case $E<M$, showing a Cauchy surface across region $\mathrm{I}$.

we find $\mathrm{d} R / \mathrm{d} v$ along $u=$ constant rays (outgoing rays). It is easy to see that

$$
\frac{\mathrm{d} R}{\mathrm{~d} v}=\frac{P(R)}{2} \text {. }
$$

Evaluating this as $R \rightarrow 0$, we find that it diverges positively independent of $E$ and $M$. Thus the Reissner Nordström singularity is always locally naked.

It is useful to cast the initial conditions in terms of $p_{r}$ and $2 m$ instead of $E$ and $M$.

$$
E^{2}=R\left(t_{\text {in }}, r\right)^{4} p_{r}\left(t_{\text {in }}, r\right)
$$

and

$$
2 M=2 m\left(t_{\text {in }}, r\right)-R\left(t_{\text {in }}, r\right)^{3} p_{r}\left(t_{\text {in }}, r\right) .
$$

Thus depending on how $2 m\left(t_{\text {in }}, r\right)$ the initial mass (at any $r$ ) compares with the radial pressure dependent quantity $R\left(t_{\text {in }}, r\right)^{3} p_{r}\left(t_{\text {in }}, r\right)+2 R\left(t_{\text {in }}, r\right)^{2} \sqrt{p_{r}\left(t_{\text {in }}, r\right)}$, one has eventually either a naked or a covered singularity.

Further, we examine the nature of initial data for regularity. We need to consider the behaviour of $R\left(t_{\text {in }}, r\right)$, for this purpose. We use equation (27) and the monotonicity of the right-hand side of (23). In the case $E \leqslant M, R\left(t_{\text {in }}, r\right)$ does not vanish at any $r$ and hence $p_{r}$ as well as $\rho$ do not diverge anywhere on the initial Cauchy surface. The same is not true however in the case $E>M$. We know that the singularity is met by all Cauchy surfaces as pointed out in the previous section. $R\left(t_{\text {in }}, r\right)$ vanishes at $(t=0, r=0)$. Using (27) and (23), we find that $R\left(t_{\text {in }}, r\right)$ vanishes at points on each surface of constant positive $t$. Since the surfaces begin from $t=0$ with increasing $t, p_{r}$ as well as $\rho$ diverge at a point on the surface $t=0$ considered as initial (and on the subsequent surfaces also).

The singularity develops eventually and a Cauchy horizon forms (illustrated in figure 1).

\section{Quantum stress tensor for the Reissner Nordström solution}

We now obtain the quantum stress tensor for the Reissner Nordström case $E<M$. It should be noted here that we employ the Wald axioms all over the region-when actually, we only 
demand that one begins with a state of the Hadamard form near the initial Cauchy surface. This supports the suggestion that the divergence on the Cauchy horizon we obtain is not an artefact of assumptions on the quantum stress tensor prescription or on the state, made locally. One could certainly work out the same expressions using the standard coordinates as done by Hiscock, under local validity of the Wald axioms. Nevertheless, we use the coordinate system in the previous section. Thus, we work explicitly with coordinates covering the Cauchy development and implicitly require the Hadamard property assumption only on the initial Cauchy surface.

We begin with the explicit expression of the line element in the $(t, r)$ coordinates, i.e. in the form (22),

$$
\mathrm{d} s^{2}=\frac{P(R)}{1-g^{2}(R)} \mathrm{d} t^{2}-\frac{g^{2}(R) P(R)}{1-g^{2}(R)} \mathrm{d} r^{2}-R^{2} \mathrm{~d} \Omega^{2}
$$

where

$$
P(R)=1-2 M / R+E^{2} / R^{2} .
$$

Since we investigate regions near the Cauchy horizon $R=R_{-}$, we use the fact that $g=g_{1}$. We suppress the angular part and obtain the expressions for the quantum stress tensor using the results (1) mentioned earlier. We cast the resulting $2 \mathrm{~d}$ metric in the double null form

$$
\mathrm{d} s^{2}=C^{2}(u, v) \mathrm{d} u \mathrm{~d} v
$$

where $u=t-g_{1} r, v=t+g_{1} r$ and

$$
C^{2}(u, v)=P(R) /\left(1-g_{1}^{2}\right) .
$$

The expressions for the quantum stress tensor can be easily worked out using (1)

$$
\begin{aligned}
& \left\langle T_{u u}\right\rangle=-1 / 12 \pi\left(\frac{g_{1}+1 / 2}{g_{1}^{2}-1}\right)^{2}\left[P^{\prime 2} / 4-P P^{\prime \prime} / 2\right]+\mathcal{A} \mathcal{A}(u) \\
& \left\langle T_{v v}\right\rangle=-1 / 12 \pi\left(\frac{g_{1}-1 / 2}{g_{1}^{2}-1}\right)^{2}\left[P^{\prime 2} / 4-P P^{\prime \prime} / 2\right]+\mathcal{B B}(v) \\
& \left\langle T_{u v}\right\rangle=\mathcal{R} / 24 \pi
\end{aligned}
$$

where the prime denotes a derivative with respect to $R$ and $\mathcal{R}$ is the Ricci scalar.

Here, the functions $\mathcal{A} \mathcal{A}$ and $\mathcal{B B}$ from (1) are chosen to be finite as in [3]. We are guaranteed that they stay finite once we choose them on a spacelike surface, in particular, the initial Cauchy surface. This is shown using a simple argument. We present that below.

Since the state is chosen to be Hadamard near the initial Cauchy surface, the Wald axioms yield finite expressions for the quantum stress tensor [8]. Then, using equation (37) evaluated near the initial surface, it can be easily checked that the functions $\mathcal{A} \mathcal{A}$ and $\mathcal{B B}$ have to be finite there. The initial Cauchy surface is $t=t_{\text {in }}$. Note that $u$ and $v$ depend only on $t$ and $r$. Specifying any function of $u$ on this initial surface would amount to specifying it completely. This is the case with any function of $v$. So the argument is complete.

Returning to equation (37), we would need to examine the behaviour of $P$. Real roots of $P(R)=0$ exist when $E \leqslant M$. It should also be noted that the metric $C^{2}$ diverges when $R=R_{-}$, the Cauchy horizon. The null coordinates need to be suitably transformed so as to ensure the regularity of the metric on the Cauchy horizon ${ }^{1}$. We then calculate the stress tensor using these coordinates.

1 This is similar to the issue of introducing Kruskal coordinates for the Schwarzschild metric to ensure regularity at the event horizon. See also [3, 16]. 
We perform the following coordinate transformation which ensures regularity of the metric:

$$
\begin{aligned}
& W=W_{0} \exp (-\tau u) \\
& Z=Z_{0} \exp (\tau v)
\end{aligned}
$$

where $\tau$ is any positive constant having physical dimension of inverse length and $W_{0}$ and $Z_{0}$ are constants of physical dimension length.

One then transforms all tensors to the $(W, Z)$ coordinate system. One can check that the metric remains non-zero and finite across the Cauchy horizon,

$$
g_{W Z}=\frac{R_{+}-R_{-}}{\tau^{2} W_{0} Z_{0} R_{-}^{2}} \exp \left(\frac{2 \tau R_{-}^{2}}{R_{+}-R_{-}}\right)+O\left(R-R_{-}\right) .
$$

The $\left\langle T_{W W}\right\rangle$ component can be easily seen to diverge positively, with the leading order behaviour $\frac{1}{R^{2}}$. One can also check this explicitly by transforming $\left\langle T_{u u}\right\rangle$ to $\left\langle T_{W W}\right\rangle$. In terms of $W$ the divergence is $\frac{1}{W^{2}}$ to the leading order in the approach to the Cauchy horizon. Similar behaviour is reported in the spherical dust case [16].

\section{Semiclassical radiation in Reissner Nordström spacetime}

We now consider the validity of the semiclassical approximation in the context of the Reissner Nordström background. If the semiclassical picture were to be followed right up to the Cauchy horizon in the Reissner Nordström background, the results would suggest an intense particle creation accompanying the naked singularity leading to a burst of energy on the Cauchy horizon. This behaviour is similar to dust as pointed out in the previous section. The semiclassical picture however can be taken to be physically appropriate only up to a limit, beyond which quantum gravity has to be invoked. At the same time, backreaction effects must also be considered. We address these issues in the Reissner Nordström case and compare it with dust. The quantum gravity limit is worked out by assuming that it is reached when curvature scales (of the central region of the dust cloud considered) reach the Planck scale [2]. This occurs one Planck length before the Cauchy horizon forms in the dust case. The Planck length cutoff is crucial for further physical interpretation for the dust case. For instance, if the cutoff had occurred much closer to the Cauchy horizon, the semiclassical calculation, which indicates the occurrence of a burst, would have been well within acceptable range of applicability (assuming that backreaction had remained negligible). This crucial feature is clearly brought out in the Reissner Nordström case.

We first show a key feature which concerns boundedness of curvature in the $E<M$ case. From equation (6), putting in the assumptions about $\mathcal{B}, A$ and $2 m_{0}$, we find that the density is given by

$$
8 \pi \rho=\frac{E^{2}}{R^{4}}
$$

and the radial pressure is given by

$$
8 \pi p_{r}=\frac{E^{2}}{R^{4}} .
$$

As expected, both diverge as the central singularity $(t=0, r=0)$ is approached. Planck scale values are reached when $t \sim-t_{\text {Planck }}$ where $t_{\text {Planck }}$ is the Planck time. The curvatures grow unboundedly as $R$ decreases to 0 . This would happen only after the curve $R=R_{-}$is crossed. That being the Cauchy horizon, one infers that the curvatures are bounded within the Cauchy 
development. This boundedness within the full Cauchy development is the key feature which makes this example special. We were able to show it explicitly by casting the region of interest in suitable coordinates enabling easier identification of the full Cauchy development in the problem.

One important consequence of this is that curvatures do not reach Planck scales in general before the Cauchy horizon. In particular, it is guaranteed if one chooses $R_{-}$to be much larger than the Planck length scale. Thus, we make a distinction between Planck curvature limit and Planck length cut-off. The latter is simply a null surface emerging out of an event one Planck length before the central singularity forms. We study the semiclassical behaviour up to the Planck length cutoff which we find similar to the dust case.

In order to find the total energy $E$ emitted to $\mathcal{I}^{+}$, one needs to integrate the power radiated up to the Planck length cutoff. We choose $\tau$ and $W_{0}$ such that $g_{W Z}$ tends to unity as $R \rightarrow \infty$.

$$
\mathcal{E}=\int_{\infty}^{t_{\text {Planck }}^{2}} P(W) \mathrm{d} W
$$

where $P$ is the power which turns out to be $\left\langle T_{W W}\right\rangle$. At this stage one can work in the full $4 \mathrm{~d}$ picture also but needs to resort to geometrical optics approximation for calculating the expression for $P$. We describe that here. Following [17] we express the radiated power ${ }^{2}$ as

$$
P(W)=\frac{\hbar}{48 \pi}\left(\frac{G^{\|}}{G^{\mid}}\right)^{2}
$$

where I denotes a derivative with respect to the argument. $G$ is determined by the centre $r=0$ being cast as the locus $v=G(W)$. This result is valid for all spherically symmetric backgrounds as the derivation involves modes passing through the centre irrespective of singularity formation at a later stage.

It can be easily checked from this definition that

$$
G(W)=-\frac{1}{\tau} \ln W / W_{0}
$$

The expression for energy radiated is thus obtained as

$$
\mathcal{E}=\frac{\hbar}{48 \pi W}
$$

If $W$ is limited to one Planck time before the Cauchy horizon at $\mathcal{I}^{+}$, then $\mathcal{E}$ can be seen to be of the order of the Planck mass.

This suggests that the data leading to a naked singularity do indeed stay largely classical until very near the Cauchy horizon. Nevertheless, a burst does occur beyond about a Planck length. In order to rule out such a burst on physical grounds, we cannot invoke the Planck curvature limit as in the dust case.

The issue of backreaction sheds light on this matter and the result is the other important consequence of the key feature of boundedness of curvature in the Reissner Nordström case.

We briefly recount the analysis for dust [18]. The energy density $\left\langle T_{\mu \nu}\right\rangle u^{\mu} u^{\nu}$ as seen by a co-moving observer moving with 4 -velocity $u^{\mu}$, represents the energy density associated with quantum fields. The classical background density is then compared with this. The background density grows unboundedly as the central singularity is approached. Especially near the centre, the background density is larger than the energy density of the scalar field, until the central singularity forms. This indicates that the backreaction is insignificant during the semiclassical evolution.

\footnotetext{
2 Assuming a conformally coupled field.
} 
The situation is different in the Reissner Nordström case. The quantum stress tensor grows unboundedly while the background density (43) and pressure (44) remain finite. It is thus impossible for the background curvatures to dominate over the quantum field energies at any event before the Cauchy horizon. It therefore turns out that the Reissner Nordström metric would be unsuitable as a background near the Cauchy horizon under considerations of backreaction.

\section{Discussion and conclusion}

The Schwarzschild solution has been cast in a suitable initial value form using co-moving coordinates [13], wherein pressure does not appear in the source term. Our transformation casts the Reissner Nordström solution in a similar form, but can be considered as collapse with non-zero pressure.

The initial radial pressure is related to $E$ and the initial mass function to $E$ as well as $M$. The case $E<M$ can be obtained from regular initial data. One may interpret that increasing the radial pressure as compared to the initial density tends to make the singularity globally naked, however the initial data in the latter case turn out to be singular.

Casting the Reissner Nordström metric in a particular coordinate system has enabled us to give an example where a naked singularity forms but curvatures are bounded within the full Cauchy development. By making the Cauchy development explicit in terms of coordinates, one can choose to work with certain states such as Hadamard states which guarantee the global validity of Wald axioms, once they are specified near the initial Cauchy surface. This provides stronger support for physical interpretation of the results obtained in the Cauchy development, in particular near the Cauchy horizon. The results on which these interpretations are based could have been drawn using the usual coordinates in which the Reissner Nordström solution is presented (with a suitable modification on the Cauchy horizon). However, a separate justification for the global validity of the Wald axioms would have to be given. We have avoided this.

The semiclassical analysis recovers Hiscock's result for $E<M$ which shows that the quantum stress tensor diverges on the Cauchy horizon, however with assumptions now made only on initial data. This indicates that the suggestion that there is a burst of energy from the singularity is actually a consequence of the choice of certain initial data.

In actual physical systems, the burst could be large but finite. To examine this further, we analyse the domain of semiclassical validity and radiation therein to conclude about the behaviour of initial data leading to naked singularities. Only one Planck mass of the mass of the classical configuration is evaporated away up to one Planck time of the classical Cauchy horizon. This behaviour is similar to the spherical dust case. We thus provide an example of an initial data configuration which collapses almost intact throughout the semiclassical domain up to the Planck length cutoff. However, in this example, the semiclassical domain extends beyond this cutoff. These two results put together support the interpretation that a sudden burst occurs near the Cauchy horizon, possibly for some initial data configurations.

The picture of semiclassical collapse in the dust model has been used to emphasize the need to invoke quantum gravity for understanding the spacetime region classically corresponding to the Cauchy development, in spherical symmetry. In the Reissner Nordström metric, on the contrary, that is not necessary. This would have been significant physically but for the backreaction factor. The latter, however, does not clearly indicate the occurrence of a burst in the Reissner Nordström case. Rather, the prominence of backreaction over the quantum energy seen near the Cauchy horizon suggests that the Reissner Nordström case appears to be unsuitable as a background in the region near the Cauchy horizon. We note here that 
physical unsuitability near the horizon is also suggested by non-local quantities or observer dependent divergences (e.g. $[4,7])$. The analysis was carried out considering backreaction of perturbations to the geometry near the horizon. However, we note that the unperturbed solution is certainly valid as a background over a period of evolution. We recover the same result comparing the quantum energies with the curvature. In fact, the solution behaves almost entirely classically throughout the evolution.

Since we have been emphasizing the region near the Cauchy horizon, a few comments on its classical nature are in order. Although the horizon will manifest as a surface generated by null rays in spacetime, the behaviour of certain quantities along geodesics shows irregularities. For a precise description see [19]. However, the horizon does not qualify as a physical barrier in the classical sense as the so-called 'singularity' there is weak in the Tipler sense (volumes stay bounded below in approach to the horizon). Thus the semiclassical results which do in fact suggest drastic physical behaviour near the horizon, assume significance.

We conclude that behaviour in spherical collapse involves the interplay of the semiclassical domain, onset of quantum gravity regime and prominence of backreaction, which need not be typical. We draw this conclusion based on a premise for initial conditions, unlike previous work. It may still be true that the contrasting physical behaviour of two kinds of initial data, indicated by spherical dust, is typical. One possible way for that to hold is the likely prominence of backreaction whenever the onset of quantum gravity is delayed, much like in the example given. It is not known if this could just be the case for backgrounds which are physically reasonable. For future work, we therefore suggest that more realistic examples be considered.

\section{Acknowledgments}

Part of this work was carried out by SB at Albert Einstein Institut, Max Planck Institut für Gravitationsphysik, Bundesrepublik Deutschland. SB wishes to acknowledges his wellwishers, especially his parents, Dr Madhavi Barve and Al Barve (deceased) for continual support.

\section{References}

[1] Bojowald Martin 2001 Phys. Rev. Lett. 865227

[2] Singh T P 2000 Conf. Proc. JGRG10 (Osaka, Japan) Sept. 2000 (Preprint gr-qc/0012087 (and references therein))

In particular, see Harada T, Iguchi H, Nakao K, Singh T P, Tanaka T and Vaz C 2001 Phys. Rev. D 64041501

[3] Hiscock W 1977 Phys. Rev. D 153054 Fulling S A 1978 Rep. Prog. Phys. 411313

[4] Hiscock W A 1981 Phys. Lett. A 83110

[5] Penrose R 1968 Battelle Rencontres ed C M DeWitt and J A Wheeler (New York: Benjamin) p 222

[6] Brady P R Proc. X Brazilian School on Cosmology and Gravitation at press

[7] Poisson E and Israel W 1990 Phys. Rev. D 411796

[8] Birrel N D and Davies P C W 1982 Quantum Fields in Curved Space (Cambridge: Cambridge University Press)

[9] Wald R M 1994 Quantum Field Theory in Curved Spacetime and Black Hole Thermodynamics (Chicago, IL: University of Chicago Press)

[10] Junker W 1996 Rev. Math. Phys. 81091

Junker W 2002 Rev. Math. Phys. 14511 (erratum)

Radzikowski M J 1996 Commun. Math. Phys. 179529

[11] Kay B S and Wald R M 1991 Phys. Rep. 20749

[12] Fulling S, Sweeny M and Wald R 1978 Commun. Math. Phys. 63257

Further refinements of the proof are also worked out based on propagation of singularities theorem in microlocal analysis-see Radzikowski M J 1996 Commun. Math. Phys. 179529

Radzikowski M J 1996 Commun. Math. Phys. 1801 
[13] Landau L D and Lifshitz E M 1976 Classical Theory of Fields (Oxford: Pergamon)

[14] Barve S, Singh T P and Witten Louis 2000 Gen. Rel. Grav. 32697

[15] Singh T P and Joshi P S 1996 Class. Quantum Grav. 13559

[16] Barve S, Singh T P, Vaz C and Witten L 1998 Phys. Rev. D 58104018

[17] Ford L H and Parker L 1978 Phys. Rev. D 171485

[18] Iguchi H and Harada T 2001 Class. Quantum Grav. 183681

[19] Burko L M and Ori A 1997 Phys. Rev. D 567820

Burko L M 1997 Phys. Rev. Lett. 794958

Burko L M 1999 Phys. Rev. D 60104033 